\title{
Classical Solutions of the Chiral Model, Unitons, and Holomorphic Vector Bundles
}

\author{
R. S. Ward \\ Department of Mathematical Sciences, University of Durham, South Road, Durham DH1 3LE, \\ England
}

\begin{abstract}
This paper deals with classical solutions of the $S U(2)$ chiral model on $\mathbb{R}^{2}$, and of a generalized chiral model on $\mathbb{R}^{2+1}$. Such solutions are shown to correspond to certain holomorphic vector bundles over minitwistor space. With an appropriate boundary condition, the solutions (called 1-unitons in [9]) correspond to bundles over a compact 2-dimensional complex manifold, and the problem becomes one of algebraic geometry.
\end{abstract}

\section{Introduction}

Minitwistor space $T \mathbb{P}_{1}$ is a 2-dimensional complex manifold which was used by Hitchin $[5,6]$ in the construction of monopoles on 3-dimensional Euclidean space. The solutions of the Bogomolny equations for monopoles on $\mathbb{R}^{3}$ correspond to certain holomorphic vector bundles over $T \mathbb{P}_{1}$. However, by imposing a different "reality" condition on such vector bundles, one can generate the solutions of the hyperbolic version of the Bogomolny equations, i.e. solutions which live on $(2+1)$ dimensional space-time $\mathbb{R}^{2+1}$. These equations form an integrable hyperbolic system, and they include, as special cases, such well-known soliton equations as the sine-Gordon, Korteweg-de Vries and nonlinear Schrödinger equations. So solutions of these correspond to holomorphic vector bundles over $T \mathbb{P}_{1}$; see [12] for more details.

The purpose of this paper is to deal with holomorphic vector bundles which extend to a certain compactification $\mathbb{T}$ of $T \mathbb{P}_{1}$. This excludes, for example, those bundles which correspond to soliton solutions of the sine-Gordon equation. But it turns out to be the right sort of boundary condition for the chiral model in $2+1$ dimensions. The hyperbolic Bogomolny equations referred to above can be rewritten as a chiral equation with torsion term, and the aim in this paper is to describe how solutions of this chiral equation correspond to (and can be generated from) vector bundles over $T \mathbb{P}_{1}$ or $\mathbb{T}$.

A chiral field is a map from $\mathbb{R}^{2+1}$ into a Lie group $G$, satisfying a certain nonlinear equation. In the case of static (time-independent) fields, one has a map 
from $\mathbb{R}^{2}$ into $G$, and then the chiral equation is just the condition that this map be harmonic. Harmonic maps from $\mathbb{R}^{2}$ into Lie groups have been extensively studied; in particular, the paper [9] gives a comprehensive treatment of harmonic maps into $U(N)$. It introduces the idea of $n$-unitons, which for $n=1,2, \ldots$ provide a hierarchy of families of solutions.

It turns out that for a given value of $N$, the only $n$-unitons that matter have $1 \leqq n$ $\leqq N-1$. We shall only deal with the case $N=2$, and therefore only with 1-unitons; but it seems likely that the results can be extended to $n>1$.

The paper [9] approaches the problem as one of analysis, and part of the purpose of the present paper is to show how this material can be translated into complex-analytic geometry and algebraic geometry. It also provides an alternative view of a well-known method of generating certain soliton solutions, sometimes called the "Riemann problem with zeros" [8].

The necessary parts of [9] are reviewed in Sect. 2. In Sect. 3 we see how solutions of the chiral model on $\mathbb{R}^{2+1}$ correspond to holomorphic vector bundles over minitwistor space $T \mathbb{P}_{1}$. The vector bundles which generate static 1-unitons are more special still; they are constructed in Sect. 4. One is led to a description of the bundle in terms of a "meromorphic framing," which generalizes the way in which a meromorphic section of a line bundle determines that bundle (because it determines a divisor).

The static 1-uniton contains an arbitrary meromorphic function of one complex variable. If (and only if) this function is rational, then the field has finite action. [This corresponds to having a harmonic map from $S^{2}$ to $S U(2)$.] In this case, the vector bundle extends to a compactification $\mathbb{T}$ of $T \mathbb{P}_{1}: \mathbb{T}$ is a (compact) rational ruled surface. This is discussed in Sect. 5. The singularities of the meromorphic framing correspond to jumping lines of the bundle in $\mathbb{T}$. There are analogies with various other "twistor" constructions: for example, monopoles can also be parametrized by rational functions [3], and Yang-Mills instantons on $S^{4}$ correspond to holomorphic vector bundles over a rational ruled surface (not the same one as $\mathbb{T})[2,1,7]$.

The above is then generalized to the non-static case; this is dealt with in Sect. 6. These more general bundles give rise to solutions which can be interpreted as moving multi-soliton solutions in $2+1$ dimensions [10]. Many questions and open problems remain: a few of these are listed in Sect. 7.

\section{Harmonic Maps into $S U(2):$ The Uniton}

This section is a brief summary of the relevant results of [9]. It deals with harmonic maps from $\Omega$ into the unitary group $U(N)$, where $\Omega$ is a simply-connected domain in $\mathbb{R}^{2}$.

If $J=J(x, y)$ is a map into $U(N)$, then the harmonic equations are

$$
\partial_{x}\left(J^{-1} \partial_{x} J\right)+\partial_{y}\left(J^{-1} \partial_{y} J\right)=0
$$

Here $\partial_{x}$ denotes $\partial / \partial x$ etc. If we define null coordinates $z$ and $\bar{z}$ by $z=x+i y$ and write $\partial=\partial / \partial z$, then (1) becomes

$$
\bar{\partial}\left(J^{-1} \partial J\right)+\partial\left(J^{-1} \bar{\partial} J\right)=0 .
$$


This equation is "completely integrable," in the sense that it can be written as the compatibility condition for a pair of linear equations involving a "spectral parameter" $\lambda[4,8]$. Define

$$
A_{z}=\frac{1}{2} J^{-1} \partial J, \quad A_{\bar{z}}=\frac{1}{2} J^{-1} \bar{\partial} J .
$$

Then the pair of linear operators

$$
\bar{\partial}+(1-\lambda) A_{\bar{z}}, \quad \partial+\left(1-\lambda^{-1}\right) A_{z}
$$

commute for all $\lambda \in \mathbb{C}^{*}=\mathbb{C}-\{0\}$, as a consequence of (2). Conversely, if (4) commute for all $\lambda$, then there exists a $J$ satisfying (3) and (2).

Given that (4) commute, one can find a solution $E_{\lambda}$ of the equations

$$
\bar{\partial} E_{\lambda}=(1-\lambda) E_{\lambda} A_{\bar{z}}, \quad \partial E_{\lambda}=\left(1-\lambda^{-1}\right) E_{\lambda} A_{z} .
$$

Theorem [9]. If $J: \Omega \rightarrow U(N)$ is harmonic, then there exists a solution $E_{\lambda}: \Omega \times \mathbb{C}^{*}$ $\rightarrow G L(N, \mathbb{C})$ of $(5)$, holomorphic for $\lambda \in \mathbb{C}^{*}$ and real-analytic for $(z, \bar{z}) \in \Omega$, such that $E_{-1}=J$ and

$$
\left(E_{\bar{\lambda}-1}\right)^{*}=\left(E_{\lambda}\right)^{-1},
$$

where ${ }^{*}$ denotes complex conjugate transpose. Conversely, suppose that $E_{\lambda}$ is holomorphic on $\mathbb{C}^{*}$ and real-analytic on $\Omega$, and that the $A_{z}, A_{\bar{z}}$ defined by (5) are independent of $\lambda$. Then $J=E_{-1}$ is harmonic.

This $E_{\lambda}$ is called an extended solution corresponding to $J$. As described here, it is not quite uniquely determined by $J$, but a unique choice can be made ([9], Theorem 2.3); we do not need this here. finite.

One can expand $E_{\lambda}$ in a Laurent series in $\lambda$. In simple cases, this series may be

Definition. An $n$-uniton is a harmonic map $J: \Omega \rightarrow U(N)$ which has an extended solution of the form

$$
E_{\lambda}=\sum_{k=0}^{n} T_{k} \lambda^{k},
$$

where the $T_{k}=T_{k}(z, \bar{z})$ are $N \times N$ matrices.

Note that a constant $J$ is a 0 -uniton (take $E_{\lambda}=J$ ), but is also an $n$-uniton for any $n$ [take $E_{\lambda}=J(-\lambda)^{n}$ ]. The uniton number can always be increased in this artificial way. But any given uniton has a minimal uniton number.

Theorem [9]. If $J: \Omega \rightarrow U(N)$ is a uniton, then its minimal uniton number is less than $N$. If $J: \mathbb{R}^{2} \rightarrow U(N)$ is harmonic, and has finite action

$$
-\int \operatorname{tr}\left(J^{-1} \partial J J^{-1} \bar{\partial} J\right) d^{2} x<\infty,
$$

then it has finite uniton number, i.e. it is an n-uniton for some $n$.

So the finite-action harmonic maps from $\mathbb{R}^{2}$ into $U(2)$ are all 1-unitons. It is easy to describe these. Let $f=f(z)$ be a meromorphic function on $\Omega$. Then

$$
E_{\lambda}=-i \mathbb{1}+\frac{i(1-\lambda)}{1+|f|^{2}}\left[\begin{array}{cc}
1 & f \\
\bar{f} & |f|^{2}
\end{array}\right]
$$


is an extended solution, and

$$
J=E_{-1}=\frac{i}{1+|f|^{2}}\left[\begin{array}{cc}
1-|f|^{2} & 2 f \\
2 \bar{f} & |f|^{2}-1
\end{array}\right]
$$

is the corresponding 1 -uniton. (Here $\mathbb{1}$ denotes the identity matrix.) Up to multiplication by constant matrices, every 1 -uniton on $\Omega$ has the form (10).

In order to get finite-action 1-unitons on $\mathbb{R}^{2}$, one must take $f(z)$ to be a rational function of $z$. In fact, the $J$ given by (10) takes values on the equator $S^{2}$ of $S U(2)$, and the condition of finite action means that $J$ extends to the conformal compactification $S^{2}$ of $\mathbb{R}^{2}$, so in effect $J$ is a harmonic map from $S^{2}$ to $S^{2}$. Such maps are necessarily rational holomorphic (or anti-holomorphic). And $f(z)$ [or $\overline{f(z)}]$ is precisely this rational map. (Note that multiplying $J$ on each side by $\left[\begin{array}{cc}0 & 1 \\ -1 & 0\end{array}\right]$ has the effect of interchanging $f(z)$ and $\overline{f(z)}$, so the holomorphic and anti-holomorphic cases are equivalent in this sense.)

\section{Holomorphic Vector Bundles over $\mathbb{T P}_{1}$}

In this section it will be shown that harmonic maps from $\mathbb{R}^{2}$ into $S U(2)$ correspond to certain holomorphic vector bundles over a 2-dimensional complex manifold $T \mathbb{P}_{1}$, the so-called "minitwistor" space, which was used in the construction of monopoles $[5,6]$. Here $T \mathbb{P}_{1}$ denotes the holomorphic tangent bundle of the Riemann sphere $\mathbb{P}_{1}$, so $T \mathbb{P}_{1}$ is fibred over $\mathbb{P}_{1}$, with each fibre being a copy of $\mathbb{C}$ (see Fig. 1). Let $\lambda$ be the standard coordinate on the base $B \cong \mathbb{P}_{1}$, i.e. $\lambda \in \mathbb{C} \cup\{\infty\}$. The two hemispheres $U=\{\lambda:|\lambda| \leqq 1\}$ and $\hat{U}=\{\lambda:|\lambda| \geqq 1\}$ cover $B$. The fibre coordinates $\eta \in \mathbb{C}$ over $U$ and $\hat{\eta} \in \mathbb{C}$ over $\hat{U}$ are patched by $\hat{\eta}=\lambda^{-2} \eta$. The holomorphic sections
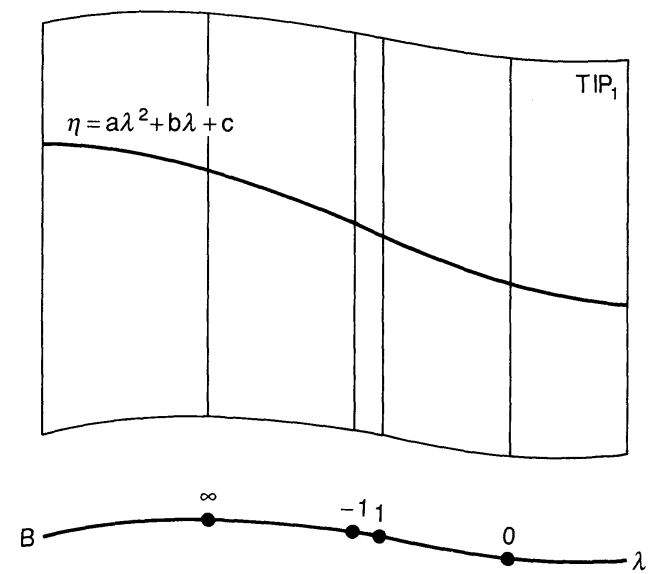

Fig. 1

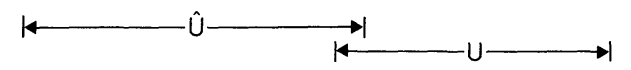


of $T \mathbb{P}_{1}$ are given by

$$
\eta=a \lambda^{2}+b \lambda+c
$$

where $a, b, c$ are complex constants. But we wish to introduce a "real structure," and restrict to real sections. This is done as follows.

Define an anti-holomorphic involution on $B$ by $\lambda \mapsto \bar{\lambda}^{-1}$ (note that this interchanges $U$ and $\hat{U}$ ). Extend this involution to $T \mathbb{P}_{1}$ by saying that the point $(\lambda, \eta)=(p, q)$ gets mapped to $(\lambda, \hat{\eta})=\left(\bar{p}^{-1},-\bar{q}\right)$. Then the real holomorphic sections, namely those preserved by the involution, are given by

$$
\eta=\bar{z} \lambda^{2}-2 i t \lambda-z
$$

where $z \in \mathbb{C}, t \in \mathbb{R}$.

Suppose now that we have a holomorphic vector bundle $E$ of rank 2 over $T \mathbb{P}_{1}$, such that

$$
\text { for every real section } \sigma,\left.E\right|_{\sigma} \text { is trivial. }
$$

We also want $E$ to be real, in the following sense. Let $F=F(\lambda, \eta)$ be the $2 \times 2$ patching matrix which patches $\left.E\right|_{U}$ to $\left.E\right|_{\hat{U}}$, i.e. $\hat{\Phi}=F \Phi$. Define $F^{\dagger}$ by

$$
F^{\dagger}(\lambda, \eta)=F\left(\bar{\lambda}^{-1},-\bar{\lambda}^{-2} \bar{\eta}\right)^{*}
$$

Then the reality condition is

$$
F^{\dagger}=F
$$

Another way of stating the reality condition on $E$ is that the involution on $T \mathbb{P}_{1}$ should lift to an anti-linear isomorphism from $E$ to its dual. Finally, we want the line bundle $\operatorname{det} E$ to be trivial, which can be achieved by imposing the condition

$$
\operatorname{det} F=1 \text {. }
$$

Such bundles [i.e. those satisfying (12), (14), (15)] correspond to $S U(2)$ YangMills-Higgs fields, satisfying the Bogomolny equations, on $(2+1)$-dimensional flat space-time. A slightly different reality structure (namely $\lambda \mapsto-\bar{\lambda}^{-1}$ ) produces a correspondence for solutions of the Bogomolny equations on 3-dimensional Euclidean space, and this was used in the construction of monopole solutions $[5,6]$. In the present case, the Bogomolny equations are hyperbolic rather than elliptic; they can be obtained by dimensional reduction of the $S U(2)$ self-dual Yang-Mills equations in $2+2$ dimensions.

The Yang-Mills-Higgs fields can be written in terms of an $S U(2)$-valued scalar field $J$ and its first derivatives, and we wish to use this alternative "chiral-model" description. Since this $J$ is obtained by integrating the Yang-Mills-Higgs field, it contains slightly more information than that field, and so it corresponds to the holomorphic vector bundle $E$ together with some additional data. This extra data consists of a holomorphic trivialization (framing) of the bundle $E$ along the two lines $\lambda=1$ and $\lambda=-1$ in $T \mathbb{P}_{1}$. See Fig. 1 . The patching matrix $F(\lambda, \eta)$, which is defined in a neighbourhood of $|\lambda|=1$, is taken to be trivial on each of these lines:

$$
F( \pm 1, \eta)=\mathbb{1} \text {. }
$$


The space-time field $J$ can be obtained from $E$ in the following way. Restrict $F$ to a real section (11). Because of (12), there exist matrices $H_{\lambda}$ and $\hat{H}_{\lambda}$, holomorphic and invertible for $\lambda \in U$ and $\lambda \in \hat{U}$ respectively (and depending real-analytically on $z, \bar{z}, t)$, which split $F$ :

$$
F\left(\lambda, \bar{z} \lambda^{2}-2 i t \lambda-z\right)=\hat{H}_{\lambda} H_{\lambda}^{-1} .
$$

Because of (15) we may take $H_{\lambda}$ and $\hat{H}_{\lambda}$ to have unit determinant. Then $J=J(z, \bar{z}, t)$ is defined by

$$
J=H_{-1}\left(\hat{H}_{1}\right)^{-1} .
$$

Remarks. $1 . J$ is well-defined. First, any change of the bundle coordinates $\hat{\Phi}, \Phi \in \mathbb{C}^{2}$ must be trivial over $\lambda= \pm 1$, in order to preserve the framing there, and so will not affect $J$. Secondly, the splitting (17) is not unique, the freedom in $H_{\lambda}$ and $\hat{H}_{\lambda}$ being

$$
H_{\lambda} \mapsto H_{\lambda} \Theta, \quad \hat{H}_{\lambda} \mapsto \hat{H}_{\lambda} \Theta,
$$

where $\Theta$ is a matrix function of $z, \bar{z}, t$. But clearly (19) does not affect $J$.

2. $J$ takes values in $S U(2)$. From (15), (17), (18) it follows that $\operatorname{det} J=1$. To prove unitarity, we can argue as follows. First, (16) and (17) give

$$
\hat{H}_{1}=H_{1}, \quad \hat{H}_{-1}=H_{-1},
$$

so that

$$
J=H_{-1}\left(H_{1}\right)^{-1} .
$$

Next, (14) and (17) give

$$
\hat{H}_{\lambda}\left(H_{\lambda}\right)^{-1}=\left(H_{\bar{\lambda}-1}\right)^{*-1}\left(\hat{H}_{\bar{\lambda}-1}\right)^{*},
$$

which implies

$$
\left(H_{\bar{\lambda}-1}\right) * \hat{H}_{\lambda}=\left(\hat{H}_{\bar{\lambda}-1}\right) * H_{\lambda} .
$$

Now the right-hand side of $(22)$ is holomorphic for all $|\lambda| \leqq 1$ and the left-hand side for all $|\lambda| \geqq 1$, so by Liouville's theorem each side is independent of $\lambda$; so we may conclude that

$$
H_{1}^{*} \hat{H}_{1}=H_{-1}^{*} \hat{H}_{-1}
$$

and hence, by (20), that

$$
H_{1}^{*} H_{1}=H_{-1}^{*} H_{-1} .
$$

From (21) and (23) we get $J^{*} J=\mathbb{1}$, as claimed.

3. $J$ satisfies a partial differential equation in $x, y, t$, generalizing the harmonic map equation (2). Define two differential operators $\delta$ and $\bar{\delta}$ by

$$
\begin{aligned}
& \delta=\partial_{z}+\frac{1}{2} i \lambda^{-1} \partial_{t}, \\
& \bar{\delta}=\partial_{\bar{z}}-\frac{1}{2} i \lambda \partial_{t} .
\end{aligned}
$$


Notice that $\delta$ and $\bar{\delta}$ annihilate the expression (11). Consequently, they also annihilate the left-hand side of (17), whence

$$
\hat{H}_{\lambda}^{-1} \partial \hat{H}_{\lambda}=H_{\lambda}^{-1} \partial H_{\lambda},
$$

with a similar expression for $\bar{\varnothing}$. Now the "Liouville" argument applied to (25) tells us that each side must be linear in $\lambda^{-1}$. For the sake of convenience, we may choose the gauge $H_{1}=1$ [i.e. we may transform as in (19) so as to ensure this]. It follows that the right-hand side of (25) has the form

$$
H_{\lambda}^{-1} \text { ð} H_{\lambda}=\left(1-\lambda^{-1}\right) A_{z},
$$

and similarly

$$
H_{\lambda}^{-1} \bar{\partial} H_{\lambda}=(1-\lambda) A_{\bar{z}},
$$

for some matrices $A_{z}, A_{\bar{z}}$ of functions of $z, \bar{z}, t$. It is clear that (26), (27) is a generalization of (5). We do not want to think of $H_{\lambda}$ as an extended solution generalizing $E_{\lambda}$, because it has the wrong singularity structure. But in the same way as before, the integrability condition for the overdetermined system (26), (27) is an equation on $J$. By putting $\lambda=-1$, we see that the relation between $J$ and $A_{z}, A_{\bar{z}}$ is [cf. (3)]

$$
A_{z}=\frac{1}{2} J^{-1}\left(\partial_{z}-\frac{1}{2} i \partial_{t}\right) J, \quad A_{\bar{z}}=\frac{1}{2} J^{-1}\left(\partial_{\bar{z}}+\frac{1}{2} i \partial_{t}\right) J .
$$

And the equation on $J$ which follows from (26), (27), (28) is

$$
\left(\eta^{\mu \nu}+\varepsilon^{\mu v \alpha} V_{\alpha}\right) \partial_{\mu}\left(J^{-1} \partial_{v} J\right)=0 .
$$

Here the indices $\mu, v, \alpha$ range over the values $0,1,2$ with $x^{0}=t, x^{1}=x, x^{2}=y$; the tensor $\eta^{\mu \nu}$ is the inverse Minkowski metric in $2+1$ dimensions, given by $\eta^{\mu \nu}=\operatorname{diag}(-1,1,1) ; \varepsilon^{\mu \nu \alpha}$ is the alternating tensor, with $\varepsilon^{012}=1$; and $V^{\alpha}$ is the constant unit vector pointing in the $x$-direction, i.e. $V^{\alpha}=(0,1,0)$.

4. Clearly (29) reduces to (1) if $J$ is independent of the time coordinate $t$. So certain special bundles give rise to the "static" harmonic maps discussed in Sect. 2. One may think of (29) as a chiral equation with torsion term. This torsion term breaks Lorentz-invariance (because of the vector $V^{\alpha}$ ). But it is necessary if one wants an equation which is completely integrable. In fact, (29) is completely integrable in $2+1$ dimensions if and only if $V^{\alpha}$ is a unit spacelike vector $[10,11]$. Notice, though, that the torsion term in effect only involves first derivatives of $J$ : Eq. (29) can also be written in the (manifestly hyperbolic) form

$$
\square J=\left(\eta^{\mu v}+\varepsilon^{\mu v \alpha} V_{\alpha}\right)\left(\partial_{\mu} J\right) J^{-1}\left(\partial_{v} J\right),
$$

where $\square=\partial_{x}^{2}+\partial_{y}^{2}-\partial_{z}^{2}$ is the wave operator.

5. As mentioned earlier, one could also talk in terms of a Yang-Mills-Higgs field satisfying the Bogomolny equations

$$
D_{\mu} \Phi=\frac{1}{2} \varepsilon_{\mu \alpha \beta} F^{\alpha \beta},
$$

where $\Phi$ is the $\operatorname{su}(2)$-valued Higgs field, $F_{\mu \nu}$ is the curvature of the $S U(2)$ gauge potential $A_{\mu}$, and $D_{\mu}=\partial_{\mu}+A_{\mu}$ is the covariant derivative. In the gauge $H_{1}=1$, the 
fields $\Phi$ and $A_{\mu}$ are given in terms of $J$ by

$$
A_{1}=-\Phi=\frac{1}{2} J^{-1} \partial_{1} J, \quad A_{0}=A_{2}=\frac{1}{2} J^{-1}\left(\partial_{0}+\partial_{2}\right) J .
$$

It is easily checked that (31) is equivalent to (29), (32). One advantage of the $J$-description is that one then has a local, conserved, positive-definite energy density, namely the usual expression [10]

$$
T_{00}=-\left(\delta_{0}^{\mu} \delta_{0}^{v}+\frac{1}{2} \eta^{\mu v}\right) \operatorname{tr}\left(J^{-1} \partial_{\mu} J J^{-1} \partial_{\nu} J\right) \text {. }
$$

By contrast, the standard Yang-Mills-Higgs energy functional is not conserved if one uses the Bogomolny equations (31); to put it another way, the one which is conserved, is identically zero for solutions of (31).

Summary. There is a mapping from

(a) holomorphic vector bundles of rank 2 over $T \mathbb{P}_{1}$, satisfying (12), (14), (15), and framed over $\lambda= \pm 1$; to

(b) real-analytic maps $J: \mathbb{R}^{3} \rightarrow S U(2)$ satisfying Eq. (29).

This can easily be extended to maps into $U(N)$ or $S U(N)$.

\section{The 1-Uniton Vector Bundle}

The 1-unitons (10) give a special family of solutions of (29), and therefore a special class of holomorphic vector bundles. The purpose of this section is to describe these in more detail.

The discussion will be local, in the following sense. The 1-uniton involves a meromorphic function $f$ on a domain $\Omega$ in $\mathbb{C}$. Let us assume, first, that $f$ is holomorphic in some neighbourhood of $z=0$ (later, we shall deal with the case when $f^{-1}$ is holomorphic). In fact, let us think of $f$ as being defined on a neighbourhood $\Omega$ of the point $z=\bar{z}=t=0$ in $\mathbb{R}^{3}$, and let $\Omega^{\prime}$ denote the corresponding region in minitwistor space. So $\Omega^{\prime}$ is an open subset of $T \mathbb{P}_{1}$ containing the curve $\eta=0$.

An extended solution $E_{\lambda}$ corresponding to $J$ satisfies

$$
\begin{aligned}
& E_{\lambda}^{-1} \text { ठ } E_{\lambda}=\left(1-\lambda^{-1}\right) A_{z}, \\
& E_{\lambda}^{-1} \bar{\partial} E_{\lambda}=(1-\lambda) A_{\bar{z}},
\end{aligned}
$$

because these are the same as (5), given that $J$ and $E_{\lambda}$ are independent of $t$. The matrix $H_{\lambda}$ of the previous section satisfies (34) as well [cf. (26), (27)], from which it follows that $H_{\lambda} E_{\lambda}^{-1}$ is annihilated by both $\partial$ and $\bar{\delta}$. This means that $H_{\lambda} E_{\lambda}^{-1}$ only depends on $z, \bar{z}, t$ through the combination (11), and so it is in effect a holomorphic matrix function of $\lambda$ and $\eta$, i.e. it is defined on an open subset of $\Omega^{\prime}$.

Given $E_{\lambda}$, we want to find a matrix $K=K(\lambda, \eta)$ such that $H_{\lambda}:=K E_{\lambda}$ is holomorphic and invertible for $|\lambda| \leqq 1$, as it should be. Note that $E_{\lambda}$ is holomorphic for $|\lambda|<\infty$, but $E_{\lambda}^{-1}$ has a pole at $\lambda=0$, since $\operatorname{det} E_{\lambda}=-\lambda$. Similarly, we want $\hat{K}(\lambda, \eta)$ such that $\hat{H}_{\lambda}=\hat{K} E_{\lambda}$ is holomorphic and invertible on $|\lambda| \geqq 1$. Then the patching matrix $F$ will be given by [cf. (17)]

$$
F=\hat{H}_{\lambda} H_{\lambda}^{-1}=\hat{K} K^{-1} .
$$


In other words, $E_{\lambda}$ determines $F$ in this way.

It is straightforward to find such $K$ and $\hat{K}$. One choice that works is

$$
K=\left[\begin{array}{cc}
\lambda^{-1} & \lambda^{-1} f(-\eta) \\
0 & -1
\end{array}\right], \quad \hat{K}=\left(K^{\dagger}\right)^{-1}=\left[\begin{array}{cc}
\lambda^{-1} & 0 \\
f^{\dagger}(-\eta) & -1
\end{array}\right] \text {. }
$$

Hence one can write out $F=\hat{K} K^{-1}=\left(K K^{\dagger}\right)^{-1}$, and check that it has all the required properties: in particular, $F^{\dagger}=F$ and $\operatorname{det} F=1$. It is not the case that $F( \pm 1, \eta)=\mathbb{1}$, but choosing $K$ to be

$$
K=\left[\begin{array}{cc}
\lambda^{-1} & \left(\lambda^{-1}-\lambda\right) f(-\eta) \\
0 & -1
\end{array}\right]
$$

would achieve this as well; for the sake of simplicity we shall stick to (36).

This description of the bundle, in terms of the patching matrix $F$, is perhaps not very illuminating. An alternative description is to observe that the columns of (36) describe a pair of meromorphic sections of the vector bundle, and that this data determines the bundle, in the same way that a meromorphic section of a holomorphic line bundle determines that bundle (because it determines a divisor).

In general, this works as follows. Let $X$ be a complex manifold, and $V$ a holomorphic vector bundle of rank $n$ over $X$. Let $\Phi$ denote a set of $n$ meromorphic sections of $V$, linearly independent almost everywhere. We could call $\Phi$ a meromorphic framing of $V$. Choose an open covering $\left\{U_{\alpha}\right\}$ of $X$, such that $\Phi$ is represented by meromorphic $n \times n$ matrices $\Phi_{\alpha}$ on $U_{\alpha}$, patched together by $\Phi_{\alpha}=F_{\alpha \beta} \Phi_{\beta}$. Then the meromorphic framing determines the bundle, by $F_{\alpha \beta}=\Phi_{\alpha} \Phi_{\beta}^{-1}$. Any other meromorphic framing will determine the same bundle, up to equivalence. One could think of the meromorphic framing as determining a "generalized divisor," which in turn determines the bundle. So the $K, \hat{K}$ given by (36) determine the vector bundle in this way.

So far, we have been assuming that the function $f$ is holomorphic. If, on the other hand, $f$ has a pole, then we can repeat the procedure using $g=1 / f$ instead of $f$. In this case, (36) is replaced by

$$
\begin{gathered}
K^{\prime}=\left[\begin{array}{cc}
\lambda^{-1} g(-\eta) & \lambda^{-1} \\
1 & 0
\end{array}\right] \text { for }|\lambda| \leqq 1, \\
\hat{K}^{\prime}=\left(K^{\prime}\right)^{\dagger-1}=\left[\begin{array}{cc}
0 & \lambda^{-1} \\
1 & -g^{\dagger}(-\eta)
\end{array}\right] \text { for }|\lambda| \geqq 1 .
\end{gathered}
$$

Finally, we can get a global description by patching everything together. In fact, if $f(z)$ is meromorphic on $\mathbb{C}$, define four patches, covering $T \mathbb{P}_{1}$, by

$$
\begin{array}{cl}
U:|\lambda| \leqq 1, & |f(-\eta)| \leqq 1, \\
\hat{U}:|\lambda| \geqq 1, & |f(-\eta)| \leqq 1, \\
U^{\prime}:|\lambda| \leqq 1, & |f(-\eta)| \geqq 1, \\
\hat{U}^{\prime}:|\lambda| \geqq 1, & |f(-\eta)| \geqq 1,
\end{array}
$$

Then $K$ on $U$, etc., is a meromorphic framing (singular along $\lambda=0$ and $\lambda=\infty$ ), which determines the holomorphic vector bundle corresponding to the 1-uniton. 
Summary. The 1-uniton extended solution $E_{\lambda}$ determines, through its singularity behaviour, a "generalized divisor" on twistor space, and this in turn determines a holomorphic vector bundle over twistor space. This can be described explicitly by means of a meromorphic framing such as (36), which has a singularity structure corresponding to that of $E_{\lambda}$.

\section{Finite-Action 1-Unitons: Bundles on a Compact Space}

In this section, it will be shown that the finite-action 1-unitons on $\mathbb{R}^{2}$ give rise to certain holomorphic vector bundles over a compact 2-dimensional complex manifold $T$. The space $T$ is a compactification of the minitwistor space $T \mathbb{P}_{1}$ used previously. Recall that $T \mathbb{P}_{1}$ is fibred over $\mathbb{P}_{1}$, with each fibre being a copy of $\mathbb{C}$. To compactify, we add on an extra section $l_{\infty}$; the resulting compact space is still fibred over $\mathbb{P}_{1}$, but each fibre is now a copy of $\mathbb{P}_{1}$.

One way of constructing $\mathbb{T}$ is to projectivize the rank 2 bundle $H^{0} \oplus H^{2}$, where $H^{n}$ denotes the $n^{\text {th }}$ power of the hyperplane-section bundle $H$ over $\mathbb{P}_{1}$. (So, in fact, $H^{0}$ is the trivial line bundle, and $H^{2}$ is the holomorphic tangent bundle.) Another way is to take a quadric cone in $\mathbb{P}_{3}$, and blow up its vertex. Either way, one obtains the rational ruled surface $\mathbb{T}$. (In algebraic geometry, this space is usually denoted $S_{2}$.)

We shall use the same coordinates $\zeta, \eta$, and $\hat{\eta}$ as before, except that now $\eta$ and $\hat{\eta}$ are allowed to take the value $\infty$ (the curve $l_{\infty}$ is given by $\eta=\infty=\hat{\eta}$ ).

A finite-action 1-uniton on $\mathbb{R}^{2}$ is determined by a rational function $f(z)$. The corresponding holomorphic vector bundle extends naturally from $T \mathbb{P}_{1}$ to $\mathbb{T}$ : we simply use the same description as before, in terms of the meromorphic framing (36), (37). So we get a holomorphic vector bundle $E$ of rank 2 over Tr.

Let us study the behaviour of this bundle in a neighbourhood of the projective line $\lambda=0$. Such a neighbourhood is covered by the two patches $U$ and $U^{\prime}$, and the patching matrix on $U \cap U^{\prime}$ which determines $E$ is

$$
K^{\prime} K^{-1}=\left[\begin{array}{cc}
f(-\eta)^{-1} & 0 \\
\lambda & f(-\eta)
\end{array}\right]
$$

It is clear from this that $\lambda=0$ is a jumping line: if $f$ has degree $n$, then the bundle restricted to the line $\lambda=\lambda_{0}$ is

$$
\left.E\right|_{\lambda=\lambda_{0}} \cong\left\{\begin{array}{lll}
H^{n} \oplus H^{-n}, & \text { if } \lambda_{0}=0 \\
H^{0} \oplus H^{0} \text { (i.e. trivial), } & \text { if } 0<\left|\lambda_{0}\right| \leqq 1 .
\end{array}\right.
$$

Such a jump is said to be of type $(n,-n)$. In the same way, $\lambda=\infty$ is also a jumping line of type $(n,-n)$.

There is an analogy here with Yang-Mills instantons [self-dual $S U(2)$ connections on $S^{4}$ ]. These correspond to "real" holomorphic vector bundles over the twistor space $\mathbb{P}_{3}$. But Donaldson [2] pointed out that framed instantons correspond to framed holomorphic vector bundles over $\mathbb{P}_{2}$, with no reality condition imposed.(Here "framed" means "framed at one point in the base space.") And Hurtubise [7] discussed these in terms of framed vector bundles over a space $\widetilde{\mathbb{P}}_{2}$ (which is $\mathbb{P}_{2}$ with one point blown up). This complex manifold $\widetilde{\mathbb{P}}_{2}$ is, like $\mathbb{T}$, a 
rational ruled surface (but it is not the same as $\mathbb{T}$ ). The basic idea in [7] is that for an instanton of charge $k>0$, there will be $k$ "vertical" jumping lines in $\mathbb{P}_{2}$ (i.e. $\lambda=\lambda_{1}, \ldots, \lambda=\lambda_{k}$ ), and each of these jumps depends on three complex parameters. So the moduli space of framed instantons has complex dimension $4 k$, as it should. The bundle over $\mathbb{P}_{2}$ is constructed by patching together "framed jumps," and this procedure serves equally well to construct bundles over $\mathbb{T}$.

The simplest example is if $J$ is a 1-uniton depending on a rational function of degree one. Such a $J$ depends on 9 real parameters [ 3 real to determine an equator in $S U(2)$, and then 3 complex to specify the map $f$ onto this equator]. The vector bundle has two jumping lines, at $\lambda=0$ and $\lambda=\infty$, each of them of type $(1,-1)$. Now the most general framed vector bundle with type $(1,-1)$ jumps at $\lambda=0, \infty$ (and with no reality condition imposed) depends on 6 complex parameters ( 3 for each jump). Imposing the reality condition reduces this to 6 real. So far, the bundle is framed at just one point, say $(\lambda, \eta)=(1,0)$; this determines a frame all along the line $\lambda=1$ (since the restriction of $E$ to this projective line is trivial). But we still have to pick a frame on $\lambda=-1$ (cf. Sect. 3), compatible with the reality condition, and this requires a further 3 real parameters, since it amounts to picking an element of $S U(2)$. So the total is 9 real parameters, as anticipated.

The situation when $f$ has degree $n \geqq 2$ is similar. The 1-uniton $J$ depends on $4 n+5$ parameters. Each of the two jumping lines is of type $(n,-n)$, and a framed jump of this type depends on $2 n+1$ complex parameters; after imposing the reality condition and picking a frame at an additional point, we get $4 n+5$ real parameters for the bundle, as expected.

Summary. A (static) 1-uniton, built from a rational function of degree $n$, corresponds to a holomorphic vector bundle over compactified minitwistor space $\mathbb{T}$. Of the one-parameter family of lines that rule $\mathbb{T}$, two are jumping lines, of type $(n,-n)$.

\section{More Bundles over $\mathbb{T}$ : Time-Dependent Unitons}

This section aims to indicate how the comments of the two previous sections can be generalized so as to apply to time-dependent 1 -unitons. So here we are dealing with maps $J: \mathbb{R}^{3} \rightarrow S U(2)$ satisfying (29). An extended solution corresponding to such a $J$ is a $G L(2, \mathbb{C})$-valued function $E_{\lambda}$ satisfying

$$
\text { б } E_{\lambda}=\left(1-\lambda^{-1}\right) E_{\lambda} A_{z}, \quad \bar{\varnothing} E_{\lambda}=(1-\lambda) E_{\lambda} A_{\bar{z}},
$$

where $A_{z}$ and $A_{\bar{z}}$ are defined by (28), and where $E_{-1}=J$. Also, $E_{\lambda}$ should satisfy the unitarity condition (6), and $E_{\lambda}$ and $E_{\lambda}^{-1}$ should be holomorphic in $\lambda$ except at isolated points. If all the singularities of $E_{\lambda}$ are simple poles lying outside the unit circle $|\lambda|=1$, and all the singularities of $E_{\lambda}^{-1}$ are simple poles lying inside $|\lambda|=1$, then $J=E_{-1}$ is a generalized 1-uniton. (For the static 1-uniton dealt with before, $E_{\lambda}$ has a simple pole at $\lambda=\infty$ and $E_{\lambda}^{-1}$ a simple pole at $\lambda=0$.)

This structure is one that has been used for generating solutions of various integrable systems of partial differential equations, and which is sometimes referred to as the "Riemann problem with zeros" $[8,4]$. The corresponding holomorphic vector bundles over minitwistor space $T \mathbb{P}_{1}$ can be constructed as in 
Sect. 4, in terms of a meromorphic framing determined by $E_{\lambda}$. The singularities of the meromorphic framing are located on the lines $\lambda=\lambda_{\alpha}$ where $E_{\lambda}$ or $E_{\lambda}^{-1}$ are singular. (It might be remarked that in the case of monopoles [4], the singularities lie on curves in $T \mathbb{P}_{1}$ that are not of the form $\lambda=$ constant; such bundles do not extend to the compactification $\mathbb{T}$.)

The expression for $E_{\lambda}$ is derived, for example, in [10] [which uses a different notation: $E_{\lambda}$ is replaced by $\psi=E_{\lambda}^{-1}$, and $\lambda$ by $\left.\zeta=i(1+\lambda) /(1-\lambda)\right]$. Translated into the present notation, the result is as follows.

Let $v_{\alpha}(\alpha=1, \ldots, k)$ be complex numbers with $\left|v_{\alpha}\right|<1$ (these will be the poles of $E_{\lambda}^{-1}$ ). For each $\alpha$, let $f_{\alpha}$ be a meromorphic function of the complex variable

$$
\omega_{\alpha}=z+2 i t v_{\alpha}-\bar{z} v_{\alpha}^{2}
$$

(i.e. $f_{\alpha}$ depends on $z, \bar{z}, t$ in this combination). Define a $k \times k$ matrix $\Gamma$ by

$$
\Gamma^{\alpha \beta}=i\left(\frac{1+\bar{v}_{\alpha}}{1-\bar{v}_{\alpha}}+\frac{1+v_{\beta}}{1-v_{\beta}}\right)^{-1}\left(1+\bar{f}_{\alpha} f_{\beta}\right),
$$

and define a phase factor $p$ by

$$
p=i\left[\prod_{\alpha=1}^{k} \frac{\left(1+v_{\alpha}\right)\left(1-\bar{v}_{\alpha}\right)}{\left(1-v_{\alpha}\right)\left(1+\bar{v}_{\alpha}\right)}\right]^{1 / 2} .
$$

Then the expressions for $E_{\lambda}^{-1}$ and $J^{-1}$ are

$$
\begin{gathered}
E_{\lambda}^{-1}=p \mathbb{1}+\frac{1}{2} i p \sum_{\alpha, \beta=1}^{k} \frac{\left(1-v_{\alpha}\right)(1-\lambda)}{\left(\lambda-v_{\alpha}\right)}\left(\Gamma^{-1}\right)^{\alpha \beta}\left[\begin{array}{cc}
1 & f_{\alpha} \\
\overline{f_{\beta}} & f_{\alpha} \bar{f}_{\beta}
\end{array}\right], \\
J^{-1}=p \mathbb{1}-i p \sum_{\alpha, \beta=1}^{k} \frac{1-v_{\alpha}}{1+v_{\alpha}}\left(\Gamma^{-1}\right)^{\alpha \beta}\left[\begin{array}{cc}
1 & f_{\alpha} \\
\overline{f_{\beta}} & f_{\alpha} \bar{f}_{\beta}
\end{array}\right] .
\end{gathered}
$$

If $k=1$ and $v=0$, then $\omega=z, \Gamma=\frac{1}{2} i\left(1+|f|^{2}\right), p=i$, and (42), (43) reduce to (the inverses of) (9), (10).

If $f_{\alpha}$ is a rational function of $\omega_{\alpha}$ (for each $\alpha$ ), then the total energy of the field is finite. When one plots the energy density (33), one sees that the solution (43) represents a multi-soliton configuration [10]. For each value of $\alpha$, there are $\left(\operatorname{deg} f_{\alpha}\right)$ solitons, all moving at the same constant velocity (determined by $v_{\alpha}$ ). When an $\alpha$-soliton and a $\beta$-soliton pass by each other, no scattering occurs.

It is worth emphasizing that the values of this general time-dependent solution do not lie on a Grassmannian submanifold $\mathbb{P}_{1}$ of $S U(2)$ (unlike in the static case, where they do).

In the same way as in Sects. 4 and 5, one can describe the holomorphic vector bundle corresponding to (42), (43) in terms of a meromorphic framing, and (for $f_{\alpha}$ rational) these bundles extend to the compactified space $\mathbb{T}$. The lines $\lambda=v_{1}, \ldots, v_{k}$, $\bar{v}_{1}^{-1}, \ldots, \bar{v}_{k}^{-1}$ are jumping lines. Let us content ourselves here with considering the parameter count in the case where each $f_{\alpha}$ has degree one (so there are $k$ "simple" solitons, each moving with a different velocity). To specify the solution $J$ one has to give the $k$ complex numbers $v_{\alpha}$, the $k$ functions $f_{\alpha}$, and an overall constant $S U(2)$ rotation: this adds up to $8 k+3$ real parameters. On the other hand, a framed bundle with $2 k$ jumps, each of type $(1,-1)$, depends on $8 k$ complex parameters; 
after imposing the reality condition and framing at an additional point, we also get $8 k+3$ real parameters.

It is amusing to note that if we omit the framing, which amounts to dealing with Yang-Mills-Higgs fields satisfying (31) rather than chiral fields satisfying (29), then the number of parameters is $8 k-3$, which is the same as that of $S U(2)$ Yang-Mills instantons (cf. $[1,2,7]$ ). However, it is important to emphasize that the set of all finite-energy solutions of (29) is infinite-dimensional. The instanton problem is elliptic, and there the condition of finite energy is sufficient to make the solution space finite-dimensional. That is not the case with the hyperbolic equation (29); see item (2) in the next section.

\section{Some Open Problems}

There are a number of open problems and questions that should be investigated further.

1. In Sect. 3 it was shown that there is a mapping from bundles on $T \mathbb{P}_{1}$ to solutions of (29) on $\mathbb{R}^{3}$. Is this mapping one-to-one and onto? The problem is more delicate than that of, say, monopoles $[5,6]$, because of the different reality structure.

2. What do bundles over the compact space $\mathbb{T}$ correspond to in space-time? In other words, what are the boundary conditions that one must impose on $J$, to ensure that solutions of (29) with these boundary conditions correspond to bundles over $\mathbb{T}$ ? As pointed out in Sect. 6, finite energy is not enough. The condition that seems to be involved is that $J$ should extend across $t=\infty$. More precisely, if we take any timelike line in $\mathbb{R}^{2+1}$ and identify $t=-\infty$ with $t=+\infty$ to form a circle, then $J$ should be real-analytic on this circle. In this context, it might be pointed out that points in $T \mathbb{P}_{1}$ with $|\lambda| \neq 1$, are in one-to-one correspondence with oriented timelike lines in $\mathbb{R}^{2+1}$. The real sections through $Z$ and $\bar{Z}$ in $T \mathbb{P}_{1}$ correspond to the points on the timelike line (see Fig. 2).

3. The bundle on $\mathbb{T}$ has one topological invariant, namely its second Chern number. This will correspond to a conserved topological charge in space-time; what exactly is it?
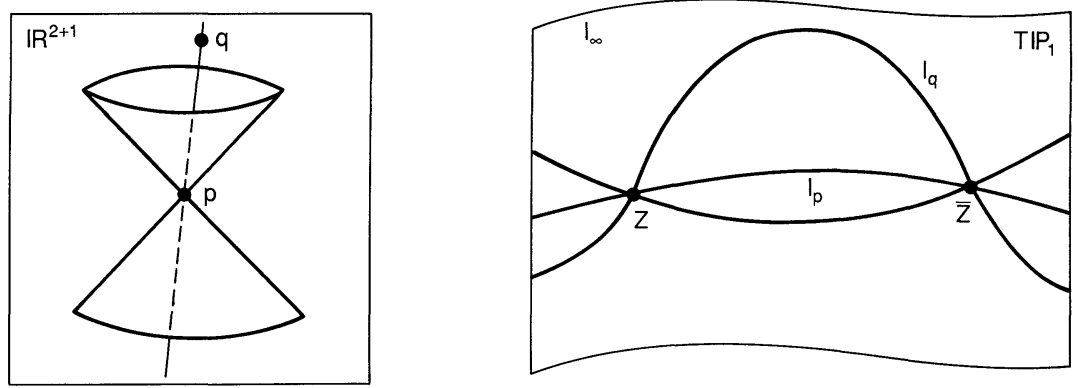

Fig. 2 
4. This paper has dealt with 1-unitons and generalized 1-unitons, and with the gauge group $S U(2)$. With larger gauge groups, one is led to $n$-unitons, with $n>1$. These give rise to bundles over $T \mathbb{P}_{1}$, of rank greater than two. Do these bundles extend to the compactified space $\mathbb{T}$ ?

Acknowledgement. I am grateful to the Durham University Research Foundation for research support.

\section{References}

1. Atiyah, M.F.: Instantons in two and four dimensions. Commun. Math. Phys. 93, 437-451 (1984)

2. Donaldson, S.K.: Instantons and geometric invariant theory. Commun. Math. Phys. 93, 453-460 (1984)

3. Donaldson, S.K.: Nahm's equations and the classification of monopoles. Commun. Math. Phys. 96, 387-407 (1984)

4. Forgács, P., Horváth, Z., Palla, L.: Solution-generating technique for self-dual monopoles. Nucl. Phys. B 229, 77-104 (1983)

5. Hitchin, N.J.: Monopoles and geodesics. Commun. Math. Phys. 83, 579-602 (1982)

6. Hitchin, N.J.: On the construction of monopoles. Commun. Math. Phys. 89, 145-190 (1983)

7. Hurtubise, J.: Instantons and jumping lines. Commun. Math. Phys. 105, 107-122 (1986)

8. Novikov, S., Manakov, S.V., Pitaevskii, L.P., Zakharov, V.E.: Theory of Solitons, Chap. III. New York: Plenum Press 1984

9. Uhlenbeck, K.K.: Harmonic maps into Lie groups (Classical solutions of the chiral model). J. Diff. Geom. 30, 1-50 (1989)

10. Ward, R.S.: Soliton solutions in an integrable chiral model in $2+1$ dimensions. J. Math. Phys. 29, 386-389 (1988)

11. Ward, R.S.: Integrability of the chiral equations with torsion term. Nonlinearity $1,671-679$ (1988)

12. Ward, R.S.: Twistors in $2+1$ dimensions. J. Math. Phys. 30, 2246-2251 (1989)

Communicated by S.-T. Yau

Received January 5, 1989; in revised form July 26, 1989 